\title{
First-Principles Calculation of Photoabsorption Spectra of Cadmium Selenide Clusters
}

\author{
Momoko Nagaoka $^{1, *}$, Soh Ishii ${ }^{1}$, Yoshifumi Noguchi ${ }^{2}$ and Kaoru Ohno ${ }^{1}$ \\ ${ }^{1}$ Department of Physics, Yokohama National University, Yokohama 240-8501, Japan \\ ${ }^{2}$ Computational Materials Science Center, National Institute for Materials Science, Tsukuba 305-0047, Japan
}

Photoabsorption spectra of magic-number cluster, $(\mathrm{CdSe})_{34}$, in a pearl-necklace geometry are calculated for the first time by a firstprinciples approach based on the density functional theory. Photoabsorption spectra for the $(\mathrm{CdSe})_{34}$ isolated-cage geometry is also calculated. We confirmed the size dependence by comparing them with our former result of smaller magic-number cluster, $(\mathrm{CdSe})_{13}$. Three different types of supercells are supposed to simulate the pearl-necklace geometry and a double-peak structure is found in a particular supercell. We found that the cluster geometry in this supercell is the most stable among the three. [doi:10.2320/matertrans.MB200825]

(Received June 2, 2008; Accepted August 5, 2008; Published October 3, 2008)

Keywords: First-principles, CdSe, photoabsorption spectra

\section{Introduction}

Electronic structures of nano-sized clusters have attracted considerable interest for their strong size dependence, yet CdSe clusters of particularly small size had not been discovered experimentally. In a recent experimental and theoretical study by Kasuya et al., ${ }^{1)}$ however, smaller $(\mathrm{CdSe})_{n}$ clusters are found to exhibit magic number behavior at $n=13$ and 34 . The stability arises from the novel "corecage" structure as calculated by the first-principles approach. The atoms constituting this structure form a cage-like network and a couple of "core" atoms inside are linked to the cage. Noguchi et al. ${ }^{2)}$ and, more recently, Botti and Marques $^{3)}$ calculated the photoabsorption spectra for the core-cage clusters and wurtzite $(\mathrm{CdSe})_{n}$ clusters of 10-100 atoms, using the first-principles approach. Del Puerto et al. calculated the photoabsorption spectra including excitonic effects for asymmetric $\mathrm{Cd}_{n} \mathrm{Se}_{m}$ clusters of 10-80 atoms with wurtzite and zincblende structures. ${ }^{4)}$ Pradhan et al. discovered the magic number clusters making up a line like a pearlnecklace in the process of synthesis of CdSe nanowires. ${ }^{5)}$ The photoasorption spectra for this pearl-necklace geometry showed a double-peak structure. Analyzing the optical property of the CdSe cluster of different geometries by a theoretical approach is necessary to the application of the clusters as quantum dots (QDs) and arrays, such as display materials, ${ }^{6)}$ biological imaging, ${ }^{7)}$ etc.

The main purpose of this paper is to calculate the photoabsorption spectra of the pearl-necklace geometry of $(\mathrm{CdSe})_{34}$, which is one of the magic number clusters found experimentally, by using the first-principles approach based on the density functional theory (DFT) within the local density approximation (LDA). The calculation is carried out also for the isolated-cage structure of $(\mathrm{CdSe})_{34}$ and the results are compared with experiments, in particular, in the viewpoint of size dependence. Since the information about the positions of each cluster in the pearl-necklace geometry is not available from the experimental data, we assume a relatively stable geometry. From the result, we find that the

*Graduate Student, Yokohama National University double-peak structure is reproduced in the pearl-necklace geometry.

\section{Methodology}

We performed the calculation of the dielectric functions for $(\mathrm{CdSe})_{34}$ clusters of different geometries.

For the isolated-cage geometry, we supposed an FCC supercell whose size is $30 \times 30 \times 30 \AA^{3}$, fairly large compared to the diameter of a $(\mathrm{CdSe})_{34}$ cluster. For the pearlnecklace geometry, because the full relaxation of the system was not possible, we shrank just one side of a simple cubic supercell with an edge length of $25 \AA$ so as to make the distance between the clusters $3 \AA$, which is a typical value considered experimentally. Consequently, we supposed three different types of simple tetragonal supercells; the specific cell sizes are $25 \times 25 \times 14.3 \AA^{3}, 25 \times 25 \times 15.7 \AA^{3}$ and $25 \times 25 \times 16.5 \AA^{3}$. The supercells used in the pearl-necklace geometry are illustrated in Fig. 1; (a) is the case where $z$ direction is shortened and (b) is the case where $x$ direction is shortened. We used the all-electron mixed basis approach, in which LDA wavefunction is expanded in the linear combination of atomic orbitals (AOs) and plane waves (PWs). Cut-off energy for PWs is $3.98 \mathrm{Ry}$. The dielectric function was evaluated within the random phase approximation (RPA), ${ }^{8)}$

$$
\begin{aligned}
& \varepsilon(\mathbf{0}, \omega)=1-\lim _{\boldsymbol{q} \rightarrow 0} \frac{8 \pi e^{2}}{\Omega q^{2}} \\
& \times \sum_{\lambda} \sum_{\nu} \frac{\left|\left\langle\boldsymbol{q}, \lambda\left|e^{i \boldsymbol{q} \cdot \boldsymbol{r}}\right| \mathbf{0}, v\right\rangle\right|^{2}\left[f_{0}\left(\epsilon_{\boldsymbol{q}, \lambda}\right)-f_{0}\left(\epsilon_{\mathbf{0}, v}\right)\right]}{\epsilon_{\boldsymbol{q}, \lambda}-\epsilon_{\mathbf{0}, v}-\omega-i \delta}
\end{aligned}
$$

where $\Omega$ is the volume of the unit cell and $\delta$ is set to be $0.27 \mathrm{eV}$, where $1 \mathrm{eV}$ corresponds to $1.602 \times 10^{-19} \mathrm{~J}$. The photoabsorption spectra are obtained as the imaginary part of this expression.

\section{Results}

\subsection{Isolated-cage geometry}

The dotted curve is the experimental result ${ }^{1,2)}$ and the solid curve is the theoretical (calculated) result. The result for 

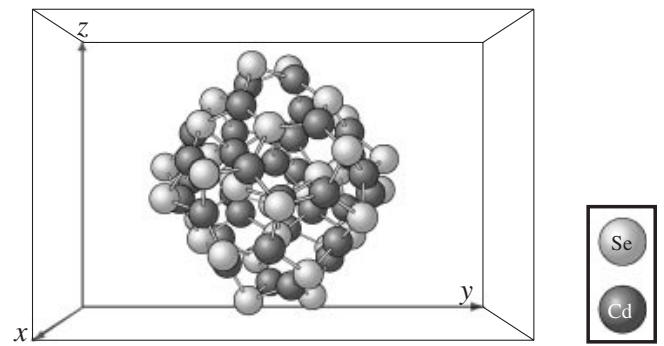

(a) Supercell where only $z$ direction is shortened.
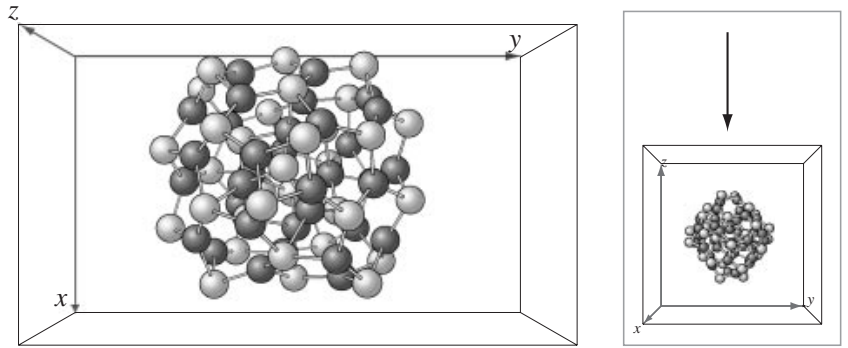

(b) Supercell where only $x$ direction is shortened, corresponding to the most stable pearl-necklace geometry.

Fig. 1 Supercells used in the calculation of pearl-necklace geometry.

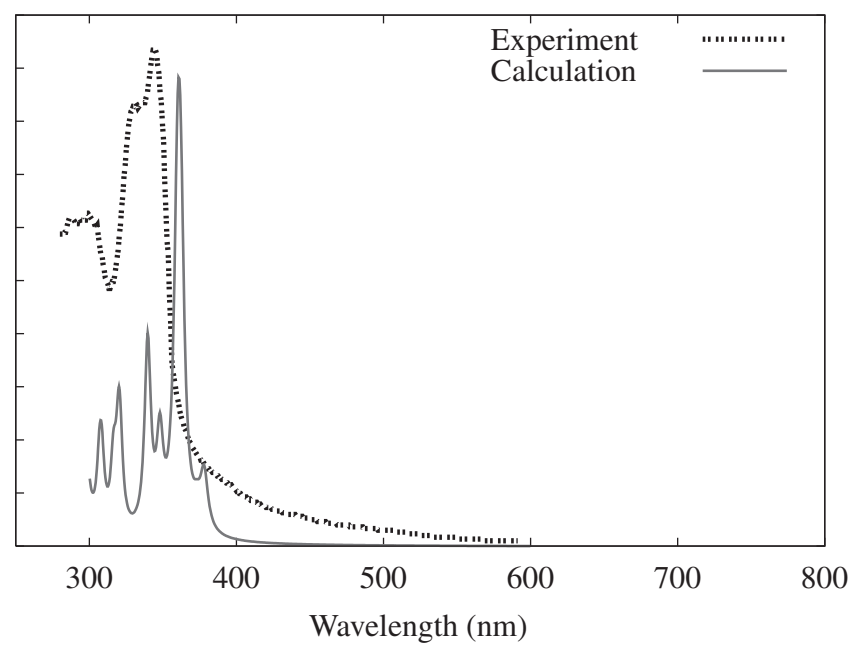

Fig. 2 Photoabsorption spectra for $(\mathrm{CdSe})_{13}{ }^{2)}$ The ordinate is an arbitrary unit.

$(\mathrm{CdSe})_{13}$ is our previous one. ${ }^{2)}$ In Figs. 2 and 3, we show the photoabsorption spectra of $(\mathrm{CdSe})_{13}{ }^{2)}$ and $(\mathrm{CdSe})_{34}$, respectively. The size dependence is evident not only in the experimental data but also in the calculated data when we compare these spectra. Although there is a discrepancy between the peak positions of the experimental and calculated results, the overall shapes of the spectra resemble each other. The calculated spectra of $(\mathrm{CdSe})_{34}$ red-shift because of the LDA. The main peak in the calculated spectra is $0.34 \mathrm{eV}$ lower than the experimental peak. Unfortunately, we do not know the experimental energy gap to be compared since there is no available photoemission data for the $(\mathrm{CdSe})_{34}$ cluster. Although the excitonic effect is not taken into account in present calculation, the binding energy of exciton is relatively small for $(\mathrm{CdSe})_{34}$ because there is enough space

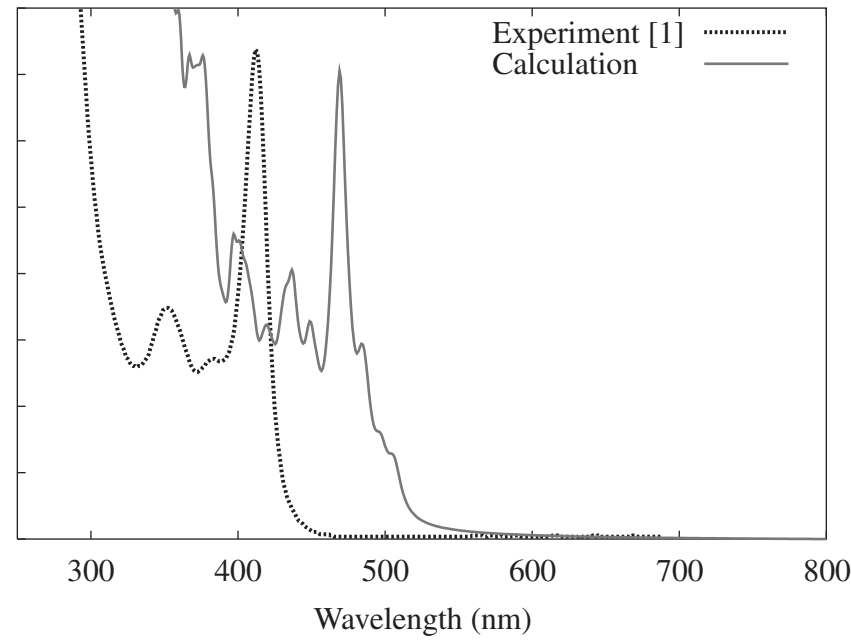

Fig. 3 Photoabsorption spectra for $(\mathrm{CdSe})_{34}$. The ordinate is an arbitrary unit.

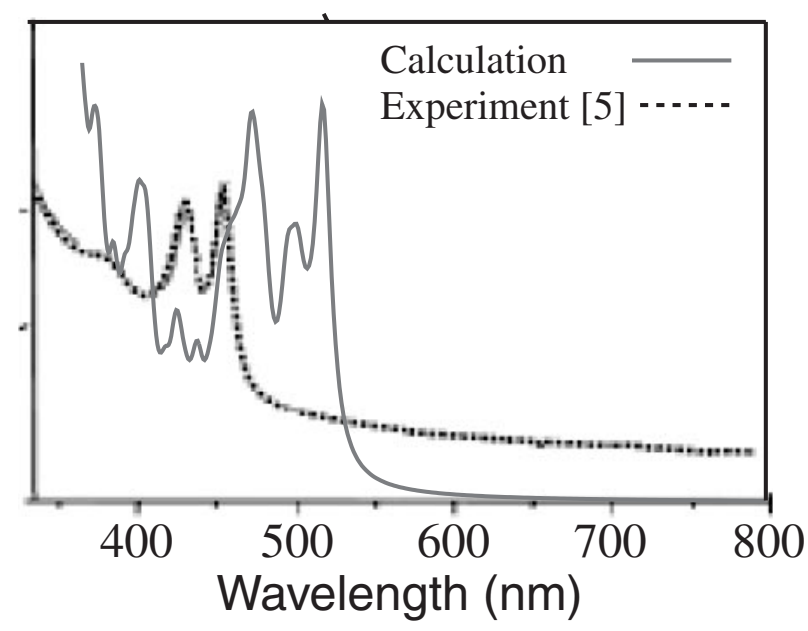

Fig. 4 Photoabsorption spectra for the $(\mathrm{CdSe})_{34}$ magic-number cluster in pearl-necklace geometry. The abscissa is the photon wavelength in units of $\mathrm{nm}$ and the ordinate is an arbitrary unit. The experimental result is taken from Ref. 5).

for the exciton to spread out inside the $(\mathrm{CdSe})_{34}$ cage. Therefore the apparent difference may be mainly attributed to the effect of the LDA. On the other hand, in the $(\mathrm{CdSe})_{13}$ cluster, the binding energy becomes larger. Together with the effect of the LDA, the calculated result of $(\mathrm{CdSe})_{13}$ only seem to fit the experimental result.

\subsection{Pearl-necklace geometry}

For the calculation of the one dimensional array system of QDs, we supposed three types of supercells as mentioned. The $x, y$ and $z$ directions are defined in order to distinguish the supercells. The orientation of the cluster in the $x y z$-coordinate is chosen such that upper and lower surfaces of the cluster in the $x$ direction become relatively flat tiled by both cadmium and selenium atoms (Fig. 1(b)), although no structural optimization is performed in this study. The solid line in Fig. 4 shows the resulting photoabsorption spectra of the $(\mathrm{CdSe})_{34}$ clusters in pearl-necklace geometry where the $x$ direction is shortened. There are two peaks at $471 \mathrm{~nm}$ and 
Table 1 Total energy of pearl-necklace geometry.

\begin{tabular}{ccc}
\hline Chain Direction & Supercell & Total Energy $(\mathrm{eV})$ \\
\hline chain in $x$ direction & $x$ direction shortened & -212.69 \\
chain in $y$ direction & $y$ direction shortened & -212.31 \\
chain in $z$ direction & $z$ direction shortened & -212.26 \\
\hline
\end{tabular}

$515 \mathrm{~nm}$. Although the calculated peaks red-shift because of the LDA, the peak width is $0.23 \mathrm{eV}$. This type of double peak structure has been observed experimentally in spectra of CdSe pearl-necklace samples ${ }^{5)}$ as plotted by a dotted line in Fig. 4.

On the contrary, the spectra of the supercell where the $y$ direction is shortened has only one peak, and the spectra of the supercell where the $z$ direction is shortened has a few relatively low peaks, although the results are not shown here.

Here, due to the instability of the cluster sensitive to the direction, the issue on configuration of atoms in the cluster must not be missed out. The cluster has selectively only selenium atoms at the outermost positions in $\pm y$ and $\pm z$ directions, while cadmium and selenium atoms spread almost flat at the surfaces in $\pm x$ direction (see Fig. 1(b)).

We calculated the total energy of all the supercells by the ultrasoft-pseudopotential program (VASP) ${ }^{9,10)}$ and the results are listed in Table 1. The supercell where the $x$ direction is shortened, i.e., the $(\mathrm{CdSe})_{34}$ chain in the $x$ direction is the most stable among the three with its total energy about $0.4 \mathrm{eV}$ lower than the other two. This is presumably due to the fact that the Coulomb repulsion in the $x$ direction is much weaker that the other two ( $y$ and $z$ ) directions.

Then the spectra of the pearl-necklace geometry is compared to that of the isolated cluster (see Fig. 5). There is a strong peak in both spectra at $2.62 \mathrm{eV}$. The peak at $2.41 \mathrm{eV}$ emerges only in the pearl-necklace geometry. For further investigation, the Kohn-Sham energy levels are fitted with a gaussian type function,

$$
f(x)=e^{-\alpha(x-a)^{2}},
$$

where $a$ is Kohn-Sham eigenvalue and $\alpha=3000$ is the fitting width. The density of states (DOS) for the system where the $x$ direction is shortened is shown in Fig. 6. It is clear from Figs. 5 and 6 that the absorption at $2.41 \mathrm{eV}$ correspond to the HOMO-LUMO transition, because the Kohn-Sham gap (difference of Kohn-Sham eigenvalues between HOMO and LUMO levels) in Fig. 6 is equal to the first peak in Fig. 5. Therefore the symmetry breaking of the wavefunction due to the adjacency of the cluster which alters the forbidden transition into an allowed transition is one possibility of this double-peak-structure.

\section{Summary}

In summary, photoabsorption spectra of $(\mathrm{CdSe})_{34}$ cluster in pearl-necklace geometry are calculated with the firstprinciples approach based on the DFT within the LDA. The spectra for the cluster in the isolated-cage geometry is also calculated and we confirmed the size-dependence of the cluster by comparing the present result to the experimental and calculated results of $\mathrm{CdSe}$ cluster with another magic

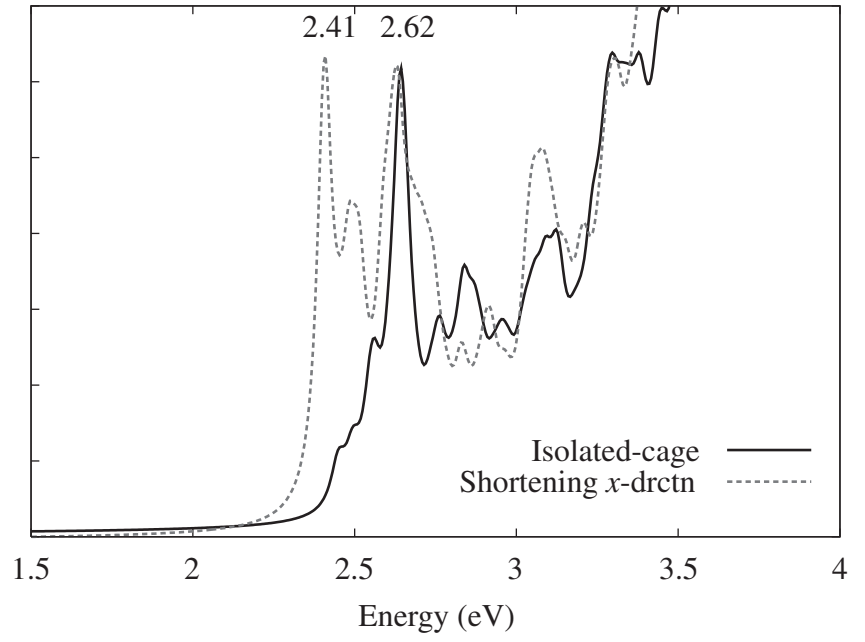

Fig. 5 Calculated photoabsorption spectra of $(\mathrm{CdSe})_{34}$ in isolated-cage and pearl-necklace geometries. The abscissa is the photon energy in units of $\mathrm{eV}$ and the ordinate is an arbitrary unit.

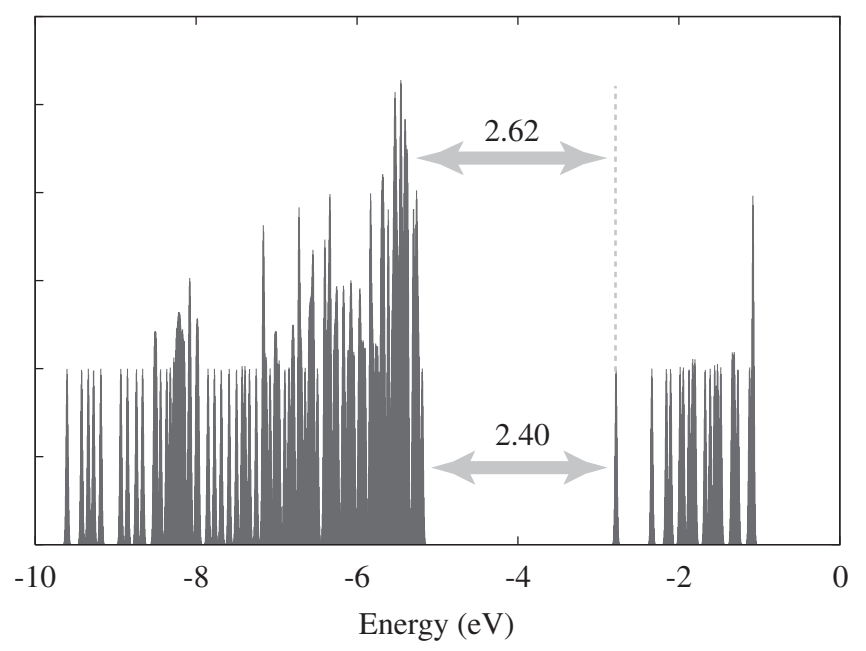

Fig. 6 The density of states of $(\mathrm{CdSe})_{34}$ in pearl-necklace geometry.

number. Although the results significantly red-shift because of the LDA, their shapes well resemble to experimental data. The results in pearl-necklace geometry vary with respect to the directions in which the clusters were approximated and we determined the most stable configuration. By comparing the present spectra in two different geometries, we found that the double-peak in pearl-necklace geometry is due to the broken symmetry of the wave functions of the cluster.

We are starting a more sophisticated calculation based on the Bethe-Salpeter equation which includes excitonic effects for some smaller clusters.

\section{Acknowledgments}

The authors would like to thank Dr. Atsuo Kasuya and Dr. Vijay Kumar for valuable discussions and data. The authors would also like to thank the Information Initiative Center at Hokkaido University for the use of the HITACHI SR11000/H1 and K1 supercomputing facilities, respectively. The present study has been partly supported by the Grand- 
in-Aid for Scientific Research in Priority Areas (Grant No. 19019006) from the Ministry of Education, Culture, Sports, Science and Technology and by the Grant-in-Aid for Scientific Research B (Grant No. 17310067) from the Japan Society for the Promotion of Science (JSPS).

\section{REFERENCES}

1) A. Kasuya et al.: Nature Mater. 3 (2004) 99.

2) Y. Noguchi, K. Ohno, V. Kumar, Y. Kawazoe, Y. Barnakov and A. Kasuya: Trans. Mater. Res. Soc. Jpn. 29 (2004) 3723.
3) S. Botti and M. A. L. Marques: Phys. Rev. B 75 (2007) 035311.

4) M. L. del Puerto, M. L. Tiago and J. R. Chelikowsky: Phys. Rev. Lett. 97 (2006) 096401.

5) N. Pradhan, H. Xu and X. Peng: Nano Lett. 6 (2006) 720.

6) W. Chan and S. Nie: Science 281 (1998) 2016.

7) R. Bakalova, H. Ohba, Z. Zhelev, M. Ishikawa and Y. Baba: Nature Bio. 22 (2004) 1360.

8) K. Ohno, K. Esfarjani and Y. Kawazoe: Computational Materials Science (Springer Series on Solid-State Science, Vol. 129) (SpringerVerlag, Berlin, Heidelberg, 1999).

9) G. Kresse and J. Furthmüller: Comput. Mater. Sci. 6 (1996) 15.

10) G. Kresse and J. Furthmüller: Phys. Rev. B 54 (1996) 11169. 